\title{
Diagnostic Trends and Clinical Characteristics of Eosinophilic Esophagitis: A Korean, Single-center Database Study
}

\author{
Ga Hee Kim, ${ }^{1,2}$ Kee Wook Jung, ${ }^{1}$ Hwoon-Yong Jung, ${ }^{\text {* }}$ Kee Don Choi, ${ }^{1}$ JungBok Lee, ${ }^{3}$ Young Soo Park, ${ }^{4}$ So-Woon Kim, ${ }^{4}$ Jeong Hoon \\ Lee, ${ }^{1}$ Do Hoon Kim, ${ }^{1}$ Ji Yong Ahn, ${ }^{1}$ Ho June Song, ${ }^{1}$ Gin Hyug Lee, ${ }^{1}$ and Jin-Ho Kim ${ }^{1}$ \\ ${ }^{I}$ Department of Gastroenterology, University of Ulsan College of Medicine, Asan Medical Center, Asan Digestive Disease Research Institute, \\ Seoul, Korea (Current address); ${ }^{2}$ Department of Health Promotion Medicine, Ewha Womans University School of Medicine, Ewha Medical \\ Research Institute, Seoul, Korea; ${ }^{3}$ Department of Clinical Epidemiology and Biostatistics, University of Ulsan College of Medicine, Asan Medical \\ Center, Seoul, Korea; and ${ }^{4}$ Department of Pathology, University of Ulsan College of Medicine, Asan Medical Center, Seoul, Korea
}

\section{Background/Aims}

The prevalence of eosinophilic esophagitis (EOE) is reportedly increasing in Western countries. However, its prevalence in Korea remains unknown. We investigated the diagnostic trends and clinical characteristics of EoE in Korea.

\section{Methods}

Using an endoscopic database maintained at a tertiary care center, we retrospectively reviewed the biopsy reports regarding 18399 biopsy specimens collected from all patients who underwent esophagogastroduodenoscopy and esophageal biopsy at this facility between 2006 and 2014. The presence of more than 15 eosinophils per high-power field with symptoms related to esophageal dysfunction was considered to indicate EoE.

\section{Results}

A total of 37 patients (male:female ratio, 29:8; mean age, $44.0 \pm 13.0$ years) were diagnosed with EoE. These patients presented with dysphagia (21.6\%), epigastric pain (21.6\%), heartburn (24.3\%), and other symptoms (32.4\%). Typical endoscopic appearance of EoE was noted in 33 cases (89.1\%) and included linear furrows in 24 cases $(64.8 \%)$, ringed esophagus in 10 cases $(27.0 \%)$, and white exudates in 11 cases (29.7\%). The median eosinophilic count was 25 per high-power field (interquartile range, 20-70). Notable histopathological findings included eosinophilic microabscesses in 21 cases (56.7\%). The diagnosis rate of EoE was found to have increased from 2006 and to 2014 ( $P$-value $<0.001$ by the Cochran-Armitage trend test).

\section{Conclusions}

The number of patients with EoE appears to have increased significantly over the 9-year period investigated, while the number of endoscopic investigations increased only marginally. Greater awareness of EoE and the role of esophageal biopsies should be considered.

(J Neurogastroenterol Motil 2018;24:248-254)

\section{Key Words}

Dysphagia; Endoscopy; Eosinophilic esophagitis; Esophagus

Received: March 28, 2017 Revised: July 28, 2017 Accepted: October 19, 2017

@ This is an Open Access article distributed under the terms of the Creative Commons Attribution Non-Commercial License (http://creativecommons. org/licenses/by-nc/4.0) which permits unrestricted non-commercial use, distribution, and reproduction in any medium, provided the original work is properly cited.

*Correspondence: Hwoon-Yong Jung, MD, PhD

Department of Gastroenterology, University of Ulsan College of Medicine Asan Medical Center, Asan Digestive Disease Research Institute, 88 Olympic-ro 43-gil, Songpa-gu, Seoul 05505, Korea

Tel: +82-2-3010-3197, Fax: +82-2-476-0824, E-mail: hyjung@amc.seoul.kr

Ga Hee Kim and Kee Wook Jung contributed equally to this work. 


\section{Introduction}

Eosinophilic esophagitis (EoE) is a clinicopathologic condition characterized by eosinophilic infiltration into the esophageal mucosa, resulting in esophageal dysfunction. ${ }^{1}$ In adults, the clinical symptoms are dysphagia, food impaction and chest pain. ${ }^{2}$ The incidence and prevalence of EoE has been increasing in Western countries where EoE has been described as a common cause of dysphagia. ${ }^{3}$ However, there are very few case reports about EoE in Asian countries. A recent study from China described the clinical manifestations and endoscopic features of EoE in 12 adult patients, and reported a prevalence of $0.34 \%$ among the general Chinese population. ${ }^{4}$ Another study, described 10 cases of EoE recorded in Japan. ${ }^{5}$ A recent study revealed that the prevalence of EoE among Korean patients with esophageal or upper gastrointestinal symptoms is $6.6 \%$. $^{6}$ However, the diagnostic trends of EoE have not been reported in Asian countries. In the current study, we reviewed an endoscopic database at a tertiary care center in order to investigate the diagnostic trends and clinical characteristics of EoE in Korea.

\section{Materials and Methods}

We performed a retrospective analysis of the endoscopic database records of adult patients (older than 18 years of age) diagnosed as having EoE by means of endoscopic esophageal biopsy performed at a single center, in Korea, between January 2006 and July 2014. The biopsy reports were searched for the term "eosinophil," and 2 endoscopists (G.H.K. and K.W.J.) reviewed the endoscopic findings of the patients that fit the search criteria. After this review of the endoscopic database, the histology slides of patients suspected of having EoE were re-reviewed by 2 highly experienced gastroin- testinal pathologists (Y.S.P. and S.Y.K.). The final analysis included patients with a peak count of 15 or more eosinophils per high -power field $(\mathrm{HPF})$ at $400 \times$ magnification and with endoscopic abnormality or esophageal dysfunction symptoms characteristic of EoE. When gastric or duodenal biopsies were included, the findings were examined to exclude co-existing gastroenteritis. Finally 37 patients were included in this study (Fig. 1). The followings clinical data were collected and analyzed: age, sex, personal history of atopic diseases (asthma, allergic rhinitis, atopic dermatitis, and food allergy), presenting symptom, diagnosis, endoscopic findings, histologic findings of esophageal biopsy, and treatment prescribed. The present study was approved by the institutional review board of the treating institute (approval number 2014-0993).

\section{Statistical Methods}

Categorical variables were analyzed using the Fisher's exact test while continuous variables were assessed using the Mann-Whitney $\mathrm{U}$ test. Continuous variables are presented as median values with the interquartile range, and categorical variables are presented as numbers with percentages. All $P$-values are two-sided, with $P<$ 0.05 indicating statistical significance.

To examine the longitudinal trends of the EoE diagnosis rate, the Cochran-Armitage trend test was used to assess the trend over 3-year intervals (2006-2008, 2009-2011, and 2012-2014). All statistical analyses were performed using SPSS version 21.0 (IBM Corp, Armonk, NY, USA).

\section{Results}

\section{Patients}

A total of 18399 endoscopic esophageal biopsies from 15598
18399 Endoscopic esophageal biopsies performed Between January 2006 and July 2014

404 Endoscopic esophageal biopsy reports mentioning the term "eosinophil"
Inclusion criteria

- Infiltration of esophageal epithelium with $\geq 15$ eosinophils/HPF

- Typical endoscopic abnormality or esophageal dysfunction symptoms
37 Patients with eosinophilic esophagitis
Figure 1. Patient selection algorithm. HPF, high power field. 
subjects were performed at our center during the study period. Among these, 404 biopsy reports mentioned the term "eosinophil." After applying the inclusion criteria, 37 patients were included in the final analysis (Fig. 1 and Supplementary Table 1). The baseline characteristics of the patients included in the study are summarized in Table 1 . The study population consisted of 29 men and 8 women (median age, 44.0 years; interquartile range for age, 32.0-53.5 years).

\section{Clinical Features}

The chief clinical manifestations of these patients were dysphagia/food impaction ( 8 cases [21.6\%]), epigastric pain ( 8 cases [21.6\%]), heartburn (9 cases [24.3\%]), and dyspepsia (3 cases [8.1\%]). The symptoms of 9 patients (24.3\%) were not evaluated.

Table 1. Clinical Features of 37 Adult Patients With Eosinophilic Esophagitis

\begin{tabular}{|c|c|}
\hline Variable & Value $(\mathrm{n}=37)$ \\
\hline \multicolumn{2}{|l|}{ Sex } \\
\hline Male & $29(78.4)$ \\
\hline Female & $8(21.6)$ \\
\hline Age (yr) & $44.0(32.0-53.5)$ \\
\hline \multicolumn{2}{|l|}{ Symptom profile } \\
\hline Dysphagia/food impaction & $8(21.6)$ \\
\hline Epigastric pain & $8(21.6)$ \\
\hline Heartburn & $9(24.2)$ \\
\hline Dyspepsia & $3(8.1)$ \\
\hline Unknown & $9(27.0)$ \\
\hline \multicolumn{2}{|l|}{ Location } \\
\hline Mid esophagus & $19(51.4)$ \\
\hline Lower esophagus & $18(48.6)$ \\
\hline Eosinophils per HPF & $25(20-71)$ \\
\hline \multicolumn{2}{|l|}{ Allergy profile } \\
\hline Asthma & $2(5.4)$ \\
\hline Allergic rhinitis & $7(16.2)$ \\
\hline Food allergy & $2(5.4)$ \\
\hline None & $26(75.0)$ \\
\hline \multicolumn{2}{|l|}{ Smoking history } \\
\hline Yes & $14(35.1)$ \\
\hline No & $23(62.2)$ \\
\hline \multicolumn{2}{|l|}{ Alcohol history } \\
\hline Yes & $15(40.5)$ \\
\hline No & $22(59.5)$ \\
\hline \multicolumn{2}{|l|}{ Blood test results } \\
\hline Tested for eosinophilia & $35(94.6)$ \\
\hline Eosinophilia ( $>500$ eosinophils $/ \mu \mathrm{L}$ ) & $8(21.6)$ \\
\hline
\end{tabular}

HPF, high power field.

Data represent total number (\%) or median (interquartile range).
Nine patients (24.3\%) had a personal history of atopy, with asthma ( 2 cases [5.4\%]), and allergic rhinitis (7 cases [16.2\%]) diagnosed most commonly. Eosinophilia was detected on at least one occasion in $8(21.6 \%)$ of 35 patients tested.

\section{Endoscopic Findings}

Typical endoscopic appearance of $\mathrm{EoE}$ was noted in 33 cases $(89.1 \%)$ and included linear furrows in 24 cases $(64.8 \%)$, ringed esophagus in 10 cases (27.0\%), white exudates in 11 cases (29.7\%), and stricture in 2 patients (5.4\%) (Fig. 2). Endoscopic findings were classified using the modified classification and grading sys-

Table 2. Endoscopic Abnormalities According to Modified Classification and Grading System in 37 Patients With Eosinophilic Esophagitis

\begin{tabular}{lc}
\hline Endoscopic findings & Value $(\mathrm{n}=37)$ \\
\hline Fixed rings & $19(51.3)$ \\
Grade 0: none & $12(32.4)$ \\
Grade 1: mild & $6(16.2)$ \\
Grade 2: moderate & 0 \\
Grade 3: severe & \\
Exudates & $17(45.9)$ \\
Grade 0: none & $18(48.6)$ \\
Grade 1: mild & $2(5.4)$ \\
Grade 2: severe & $11(29.7)$ \\
Furrows & $26(70.2)$ \\
Grade 0: absent & \\
Grade 1: present & $35(94.5)$ \\
Edema & $2(5.4)$ \\
Grade 0: absent & \\
Grade 1: present & $35(94.5)$ \\
Stricture & $2(5.4)$ \\
Grade 0: absent & \\
Grade 1: present &
\end{tabular}

Data represent the number of patients (\%) presenting with a certain endoscopic finding.

Table 3. Histologic Findings in 37 Patients With Eosinophilic Esophagitis

\begin{tabular}{lc}
\hline \multicolumn{1}{c}{ Histologic finding } & Positive/Tested \\
\hline Eosinophilic microabscess & $21 / 37$ \\
Superficial layering of eosinophils & $3 / 31$ \\
Epithelial desquamation & $21 / 37$ \\
Dilated intercellular spaces & $21 / 37$ \\
Rete peg elongation & $31 / 37$ \\
Basal zone hyperplasia & $26 / 37$ \\
Lamina propria fibrosis & $5 / 5$ \\
\hline
\end{tabular}

Values represent number of patients. 
tem in Table 2. ${ }^{7}$ The majority of biopsies were taken from the mid esophagus (19 cases [51.4\%]). Nineteen cases (48.6\%) of EoE patient's biopsies were taken from lower esophagus above $5 \mathrm{~cm}$ from Z-line. We only considered biopsies taken from at least $5 \mathrm{~cm}$ above the $\mathrm{Z}$-line.

Among a total of 37 patients with EoE, 4 patients had no EoE-specific endoscopic findings, while 33 patients had typical endoscopic findings, and all such patients were male. Among the 4 patients with no EoE-specific endoscopic findings, 2 patients had typical EoE symptoms, especially food impaction. These 2 patients (40 and 44 years of age; both male) were treated with inhalation steroids, which led to improvement of symptoms. The other 2 patients without EoE-specific findings (40 and 49 years of age; both male) underwent endoscopy to elucidate the origin of their mild symptoms such as epigastric discomfort. In these 2 patients, endoscopic findings included mucosal erosion and hyperemia with fine mucosal plaques. Unfortunately, these 2 patients were lost to follow-up.

\section{Histologic Findings}

Histopathological examination (Table 3) revealed eosinophilic infiltration of the esophageal epithelium ( $\geq 15$ eosinophils/HPF) in all patients. The median eosinophilic count was 25 per HPF (interquartile range, 20-70). Eosinophilic microabscess, defined as an aggregate of 4 or more eosinophils, was another notable histologic finding observed in 21 cases (56.7\%). Superficial layering of eosinophils, which is defined as a specific affiliation of eosinophils to aggregates in the surface layers of the epithelium was observed in 3 of 31 applicable cases. Basal zone hyperplasia (greater than $20.0 \%$ of the thickness of the epithelium) was observed in 26 cases (70.2\%). Rete peg elongation (greater than $60.0 \%$ of the total epithelial height) was observed in 31 cases $(83.7 \%)$. Finally lamina propria fibrosis epithelium was observed in 5 of 5 applicable cases.

Lamina propria fibrosis and superficial layering of eosinophils could not be evaluated in $16.2 \%$ and $86.4 \%$ of cases, respectively, as some specimens were either poorly oriented or lacked lamina propria. Figure 3 shows the typical histological features seen in our
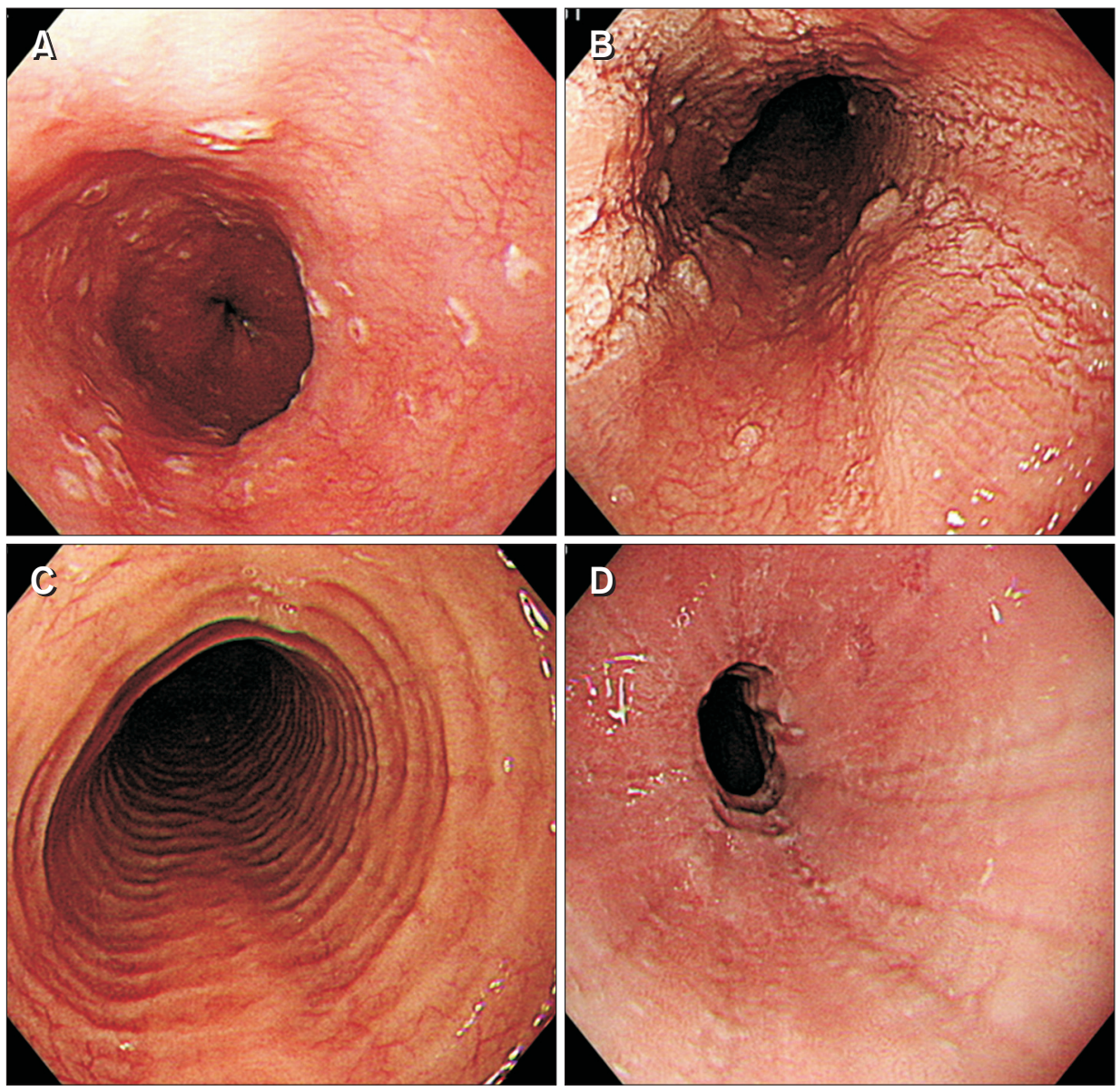

Figure 2. Endoscopic findings of eosinophilic esophagitis. (A) Whitish exudates. (B) Linear furrows with whitish exudates. (C) Ringed esophagus. (D) Stricture. 

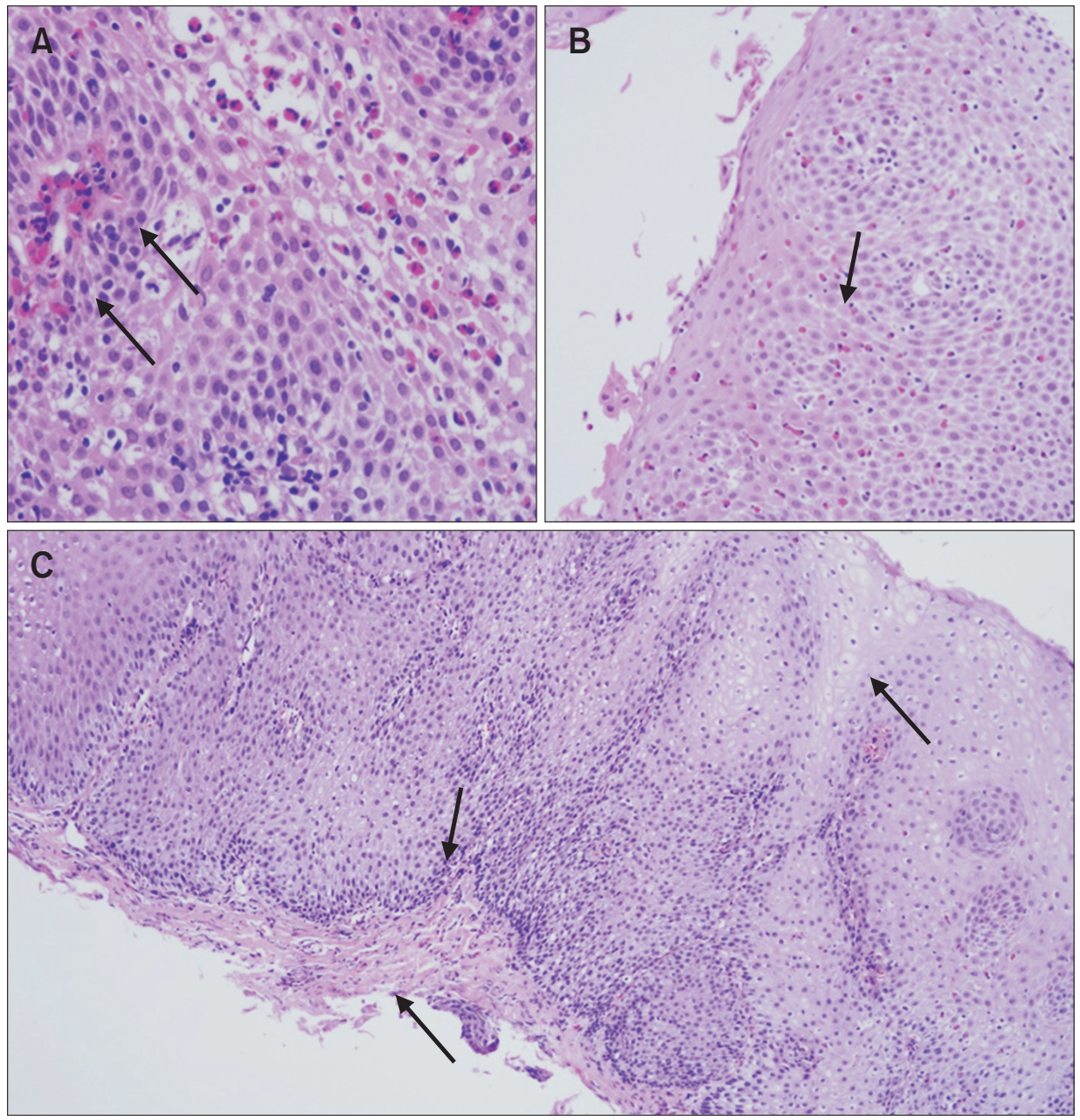

Figure 3. Histologic findings of eosinophilic esophagitis. (A) Eosinophilic microabscess $(\times 400)$. (B) Superficial layering of eosinophils $(\times 200)$. (C) Basal zone hyperplasia with rete peg elongation and lamina propria fibrosis $(\times 100)$.

EoE patients.

\section{Treatment}

Of the 37 patients included in the study, 29 (78.3\%) were treated with anti-secretory agents including proton pump inhibitors (PPIs). Among the 20 patients who did not respond to PPIs, 10 were treated with corticosteroids, which led to symptomatic relief or improvement of endoscopic findings. Follow-up esophagogastroduodenoscopy (EGD) was performed in 14 patients. Despite treatment, 5 of the 14 patients who underwent EGD still showed typical endoscopic findings (Supplementary Figure).

\section{Incidence}

Table 4 provides an overview of the number of specific procedures (ie, EGD and endoscopic esophageal biopsy) performed at our institution over the course of the nine-year study period investigated (2006-2014), as well as of the number of patients diagnosed
Table 4. Diagnostic Trend of Eosinophilic Esophagitis Over a 9-year Period Between January 2006 and July 2014

\begin{tabular}{lccc}
\hline \multirow{1}{*}{ Procedure } & \multicolumn{3}{c}{ Period } \\
\cline { 2 - 4 } & $2006-2008$ & $2009-2011$ & $2012-2014$ \\
\hline Esophagogastroduodenoscopy & 201228 & 239285 & 221455 \\
Esophageal biopsy & $5481(2.7)$ & $7003(2.9)$ & $5915(2.7)$ \\
$\begin{array}{l}\text { Eosinophilic esophagitis } \\
\text { diagnosed }\end{array}$ & 3 & 7 & 27 \\
\hline
\end{tabular}

Values represent the number of procedures. Values given in parentheses indicate the percent of patients who underwent both esophagogastroduodenoscopy and esophageal biopsy. Diagnosis rate increased significantly over the course of the study period $(P<0.001$ by the Cochran-Armitage trend test)

as having EoE by means of these procedures. To determine the variation in EoE diagnosis rate, we split the 9-year study period into 3 periods of 3 years each. The number of EGDs and esophageal biopsies appeared to have increased only slightly over the course of 
the study period. However, the number of patients with EoE appeared to have increased significantly over the 9-year period evaluated $(P<0.001$ by the Cochran-Armitage trend test).

\section{Discussion}

$\mathrm{EoE}$ is a chronic inflammatory disease restricted to the esophagus, with an increasingly higher incidence reported over the past 10 years. ${ }^{8}$ The clinical characteristics and prevalence of $\mathrm{EoE}$ have been extensively investigated in Western countries. However, in Asian countries, the disease is recognized as a rare condition and its epidemiology has not been fully characterized. The present study reviewed an endoscopic database maintained at a tertiary care center in order to investigate the diagnostic trends and clinical characteristics of $\mathrm{EoE}$ in Korea.

In Western countries, EoE was reported with a higher prevalence among middle-aged and male individuals, and it often cooccurred with allergic disease. ${ }^{9}$ These previous findings are similar to those of the present study, which showed middle-age and male predominance, but we noted less frequent comorbidity of allergic diseases (9 cases [24.3\%]). Common clinical presentations in our patients included dysphagia (8 cases [21.6\%]), epigastric pain (8 cases [21.6\%]), and heartburn (9 cases [24.3\%]). Compared to reports from Western countries, ${ }^{10}$ our study found a higher prevalence of with atypical symptoms of $\mathrm{EoE}$ (chest pain and epigastric pain similar to those noted in gastroesophageal reflux disease). However, our study does not have sufficient statistical power to claim that Korean $\mathrm{EoE}$ patients have different clinical presentations from those noted in patients from Western countries. Larger scale, nationwide studies are warranted to clarify the symptomatic characteristics of Korean EoE patients.

Typical endoscopic findings of EoE were observed in 33 cases (89.1\%), and included linear furrows in 24 cases (64.8\%), ringed esophagus in 10 cases $(27.0 \%)$, and white exudates in 11 cases (29.7\%). There were only 2 patients with strictures. These result shows some discrepancy compared with those reported in Western countries, where stricture or mucosal friability are common EoE findings. ${ }^{11}$ Bohm et al ${ }^{12}$ reported that the endoscopic findings of EoE may vary with race. In a recent Japanese study, linear furrows were more frequently noted in patients with $\mathrm{EoE},{ }^{13}$ which is similar to the findings of our present study.

We found that the number of EGD procedures increased slightly over the course of the study period, while the number of patients diagnosed with EoE increased significantly. It remains unclear whether the high prevalence of $\mathrm{EoE}$ observed in the most recent 3-year period analyzed (2012-2014) represents a real increase in the incidence of $\mathrm{EoE}$ or an increase related to enhanced awareness among endoscopists and pathologists. A population-based study in Switzerland reported an actual increase in the incidence of $\mathrm{EoE}$ and its cumulative prevalence in the past ten years. ${ }^{14}$

Several studies have reported that the prevalence of $\mathrm{EoE}$ has been increasing in Asian countries with the growing interest and awareness of this disease (Supplementary Table 2). The etiology of $\mathrm{EoE}$ is not clear. A possible link has been suggested between an immunologic response and various ingested allergens, given the high incidence of food allergies noted in these patients. In Korea, the prevalence of atopic diseases such as allergic rhinitis, atopic dermatitis, and food allergy is increasing. ${ }^{15}$ The high prevalence of atopic diseases in both pediatric and adult EoE patients suggests that EoE patients exhibit an allergic response to one or more antigens that may provoke and perpetuate this condition. ${ }^{16}$ Indeed, food allergies are known to play a substantial role in pediatric eosinophilia in Western countries. ${ }^{17}$ However, the etiology and natural history of EoE among Asian adults is not well understood. Prospective, long-term follow-up and population-based studies are warranted to clarify such aspects in EoE patients from Asian countries.

PPI-responsive esophageal eosinophilia (PPI-REE) was recently suggested to represent a disease entity distinguishable from the disease entity previously referred to as EoE. ${ }^{1}$ However, other studies suggested that PPI-REE does not differ from $\mathrm{EoE}$ in terms of either pathology or clinical manifestation. ${ }^{18}$ Therefore, PPI-REE is currently regarded as a disease entity within the disease spectrum of $\mathrm{EoE}{ }^{19} \mathrm{~A}$ recently published review concluded that it is difficult to clearly distinguish between $\mathrm{EoE}$ and PPI-REE. ${ }^{20}$ As ours was a retrospective study, we could not treat all of the study patients with PPIs. Moreover, a considerable number of patients were lost to follow-up. For this reason, we were not able to determine whether $\mathrm{EoE}$ and PPI-REE differed in terms of endoscopic or histological findings.

There are several potential limitations in our study. First, this was a retrospective study, and it was difficult to ensure that all clinical manifestations were accounted for in the reports. Furthermore, not all biopsy specimens were obtained from multiple levels of the esophagus, and eosinophilic gastroenteritis could not be excluded in all cases as biopsies of the stomach and duodenum were not routinely performed. Second, the recognition of $\mathrm{EoE}$ in Korea is still low, and therefore our sample included only a small number of cases, with inconsistent time intervals for follow-up endoscopy. Third, we could not perform an esophageal manometry study or a 24-hour $\mathrm{pH}$ monitoring study to exclude the presence of other mo- 
tility disorders and gastroesophageal reflux disease.

Our findings suggest that the number of patients with EoE increased significantly from 2006 to 2014, while the number of EGD investigations and esophageal biopsies increased only marginally. A greater awareness of $\mathrm{EoE}$ and of the role of esophageal biopsies should be considered. Large-scale studies with prospective design are required in order to validate the purported increase in the prevalence of EoE in Korea.

\section{Supplementary Materials}

Note: To access the supplementary tables and figure mentioned in this article, visit the online version of Journal of Neurogastroenterology and Motility at http://www.jnmjournal.org/, and at https:// doi.org/10.5056/jnm17047.

\section{Financial support: None.}

\section{Conflicts of interest: None.}

Author contributions: Ga Hee Kim, Kee Wook Jung, Jin-Ho Kim, and Hwoon-Yong Jung designed the study; JungBok Lee and Kee Don Choi analyzed the raw data and performed statistical analyses; Young Soo Park, So-Woon Kim, and Jeong Hoon Lee contributed to data collection; Do Hoon Kim, Ji Yong Ahn, Ho June Song, and Gin Hyug Lee interpreted the data; Ga Hee Kim and Kee Wook Jung drafted the manuscript and prepared the manuscript for submission; and all authors revised the manuscript critically for important intellectual content and gave final approval of the version to be published.

\section{References}

1. Liacouras CA, Furuta GT, Hirano I, et al. Eosinophilic esophagitis: updated consensus recommendations for children and adults. J Allergy Clin Immunol 2011;128:3-20, e26.

2. Remedios M, Campbell C, Jones DM, Kerlin P. Eosinophilic esophagitis in adults: clinical, endoscopic, histologic findings, and response to treatment with fluticasone propionate. Gastrointest Endosc 2006;63:312.

3. Ronkainen J, Talley NJ, Aro P, et al. Prevalence of oesophageal eosinophils and eosinophilic oesophagitis in adults: the population-based Kalixanda study. Gut 2007;56:615-620.
4. Shi YN, Sun SJ, Xiong LS, Cao QH, Cui Y, Chen MH. Prevalence, clinical manifestations and endoscopic features of eosinophilic esophagitis: a pathological review in China. J Dig Dis 2012;13:304-309.

5. Tomomatsu Y, Yoshino J, Inui K, et al. Clinical features of eosinophilic esophagitis: ten Japanese cases. Dig Endosc 2013;25:117-124.

6. Joo MK, Park JJ, Kim SH, et al. Prevalence and endoscopic features of eosinophilic esophagitis in patients with esophageal or upper gastrointestinal symptoms. J Dig Dis 2012;13:296-303.

7. Hirano I, Moy N, Heckman MG, Thomas CS, Gonsalves N, Achem SR. Endoscopic assessment of the oesophageal features of eosinophilic oesophagitis: validation of a novel classification and grading system. Gut 2013;62:489-495

8. Chang F, Anderson S. Clinical and pathological features of eosinophilic oesophagitis: a review. Pathology 2008;40:3-8.

9. Gonsalves N, Kahrilas PJ. Eosinophilic oesophagitis in adults. Neurogastroenterol Motil 2009;21:1017-1026.

10. Mackenzie SH, Go M, Chadwick B, et al. Eosinophilic oesophagitis in patients presenting with dysphagia--a prospective analysis. Aliment Pharmacol Ther 2008;28:1140-1146.

11. Sgouros SN, Bergele C, Mantides A. Eosinophilic esophagitis in adults: what is the clinical significance? Endoscopy 2006;38:515-520.

12. Bohm M, Malik Z, Sebastiano C, et al. Mucosal eosinophilia: prevalence and racial/ethnic differences in symptoms and endoscopic findings in adults over 10 years in an urban hospital. J Clin Gastroenterol 2012;46:567-574

13. Fujiwara Y, Sugawa T, Tanaka F, et al. A multicenter study on the prevalence of eosinophilic esophagitis and PPI-responsive esophageal eosinophilic infiltration. Intern Med 2012;51:3235-3239.

14. Giriens B, Yan P, Safroneeva E, et al. Escalating incidence of eosinophilic esophagitis in Canton of Vaud, Switzerland, 1993-2013: a populationbased study. Allergy 2015;70:1633-1639.

15. Lee S. IgE-mediated food allergies in children: prevalence, triggers, and management. Korean J Pediatr 2017;60:99-105.

16. Penfield JD, Lang DM, Goldblum JR, Lopez R, Falk GW. The role of allergy evaluation in adults with eosinophilic esophagitis. J Clin Gastroenterol 2010;44:22-27.

17. Rothenberg ME, Mishra A, Collins MH, Putnam PE. Pathogenesis and clinical features of eosinophilic esophagitis. J Allergy Clin Immunol 2001;108:891-894.

18. Moawad FJ, Schoepfer AM, Safroneeva E, et al. Eosinophilic oesophagitis and proton pump inhibitor-responsive oesophageal eosinophilia have similar clinical, endoscopic and histological findings. Aliment Pharmacol Ther 2014;39:603-608.

19. Cheng E, Souza RF, Spechler SJ. Eosinophilic esophagitis: interactions with gastroesophageal reflux disease. Gastroenterol Clin North Am 2014;43:243-256.

20. Molina-Infante J, Bredenoord AJ, Cheng E, et al. Proton pump inhibitor-responsive oesophageal eosinophilia: an entity challenging current diagnostic criteria for eosinophilic oesophagitis. Gut 2016;65:524-531. 